\title{
A case study of analysis of natural frequency variability related to manufacturing process
}

\author{
T. Uhl and W. Lisowski* \\ Department of Robotics and Mechatronics, AGH University of Science and Technology, Kraków, Poland
}

\begin{abstract}
One of the important challenges present nowadays in the automotive industry is minimizing of a car components design time. Traditional manufacturing of a prototype is usually a time and a cost consuming process. Alternatively, rapid prototyping techniques can be used in such a case. In the reported research a brake caliper was investigated, since it is an example of an element, which should have very strictly defined structural dynamic properties. As a technique of rapid prototyping of the considered caliper the 3D printing of a mould was selected. A process of the caliper casting with the use of the "prototype" mould is different than the one with the use of the metal form. Thus it is very likely that the both considered types of the caliper would possess different properties from the point of view of structural dynamics.

Structural dynamic properties can be analyzed both numerically and experimentally. Simulation of the caliper FE model with uncertain parameters was used to analyze influence of various caliper parameters on its natural frequency values. Modal testing of the caliper was performed with the aim of investigation of applicability of Experimental Modal Analysis for determination of variability of natural frequencies resulting from the manufacturing process. In the course of this research, the natural frequencies of the prototype caliper and the standard caliper were compared.
\end{abstract}

Keywords: Structural dynamics, finite element modelling, experimental modal analysis, variability of structural properties, rapid prototyping

\section{Introduction}

The aim of this investigation was to test applicability of selected numerical and experimental methods of analysis of structural dynamics properties for determination of natural frequency values in presence of variability of a considered object specimens.

Generally, the actual variability of natural frequency values of various specimens of the same object manufactured in large series is caused by: variability of material properties, limited repeatability of forming technology (accepted tolerances of machining, casting etc.), as well as by limited assembly repeatability.

Taking into account that in the automotive industry the problem of minimisation of time and costs of a product development is of great importance a disc brake caliper made of Aluminum-Silicon casting alloys was selected as an example object for investigation.

The use of aluminum-silicon alloys in the fabrication of the disc brake calipers is justified by their high strengthto-weight ratio, which increases the performance and decreases fuel consumption. The fact that the calipers are produced by casting gives a critical importance to the design and the fabrication of permanent as well as sand moulds. A new product development requires many different steps before the product can be mass manufactured. One of them is a prototype fabrication. The testing of the prototype allows the engineers to validate the design with respect to the clients' demands. For each new prototype a new mould is designed, so it is essential that the moulds are created as fast as possible and as cheap as possible. The Rapid Prototyping (RP) methods allow the fabrication of

\footnotetext{
${ }^{*}$ Corresponding author: Department of Robotics and Mechatronics, AGH University of Science and Technology, al. Mickiewicza 30, 30-059 Kraków, Poland. Tel./Fax: +48 12634 3505; Tel.: +48 12617 3511; E-mails: tuhl@agh.edu.pl, lisowski@agh.edu.pl.
} 
prototypes in a short time and at low costs. Various RP techniques exist on the market. In this research the 3D printing was used.

In this paper the difference between natural frequency values obtained in case of application of the "prototype" mould prepared with the use of the selected RP technique with respect to the ones achieved in case of application of the metal form was investigated.

The resultant variability of determination of natural frequencies during numerical simulation and experimental testing is related to the applied analysis technique.

In the course of the project the Finite Element Analysis was applied to formulate dynamic model of a caliper, in order to calculate its natural frequencies. The "prototype" mould was then designed and produced with the use of a $3 \mathrm{D}$ printer. Next the prototype caliper was cast.

Finally, the prototype and the standard calipers were subjected to Experimental Modal Analysis (EMA) - the procedure of experimental identification of a structural dynamic model $[3,5,13]$. In this research only the estimates of natural frequency were determined.

In case of investigation of variability of natural frequency values due to manufacturing process the question arose if standard EMA was appropriate for that purpose. The variability of natural frequency values identification results should be considerably low when compared to the investigated variability of natural frequencies related to the manufacturing process.

There are various sources of EMA results variability encountered in practice.

During modal experiments temperature variations, boundary conditions' changes and pre-stress changes occur. The excitation repeatability (e.g. of hammer hits) is limited. Application of various techniques and types of excitation leads to slightly different results. The repeatability of exciters' assembly and sensors' placement is limited as well. Finally there are errors made by an operator during experiment (e.g. wrong placement of sensors, wrong orientation of sensors, mismatch of sensors' placement) that influence mainly mode shapes estimation.

A variety estimation algorithms is often concurrently used during the parameter estimation procedure. Various algorithms and implementations of these algorithms lead to slightly different estimation results. Finally, subjective reasoning of an operator during data selection (choosing various frequency subranges and/or a set of signals measured only on some part of the object) and/or during estimation results selection (commonly consisting in subjective selection of poles from the stabilization diagrams [10]), as well as the change of parameters of an estimation procedure usually change the parameter estimation results too.

It should be noted that often results of inadequacy of the modal model in case of nonlinear behaviour of a tested structure is also considered in practice to be a source of variability of EMA results.

The most famous example of investigation of modal parameters variability that base on testing results of an assembled laboratory structure (GARTEUR SM-AG19) was reported in [4]. There were considered the following factors influencing modal parameters dispersion: object properties (after reassembly), selection of experimental technique (Phase Resonance or Phase Separation Method), excitation repeatability (an impact hammer), parameter estimation technique and implementation as well as an operator activity during modal experiment and parameter estimation.

For assessment of the variability of natural frequency value the relative standard deviation of natural frequency was used:

$$
D_{\sigma}=\frac{\sigma_{f}}{E_{f}}
$$

where: $E_{f}$ is a mean value of the natural frequency estimates achieved during repeated analysis and corresponding to the same mode shape, $\sigma_{f}$ is a standard deviation of the considered natural frequency estimates.

Results of investigation of repeatability of modal parameters estimation was presented in [15] basing on a set of nine modal experiments carried out on a welded car subcomponent.

In [12] there was showed a comparison of modal parameters estimated for a composite airplane fuselage panel basing on results of seven modal experiments in which various testing techniques were used.

Variability of EMA results is a common problem of its application. The best result should be somehow extracted and indicated. In case of the modal experiment it is advisable to observe strictly the testing procedure, and carry out a series of experiments instead of a single one. In case of the modal parameter estimation one should carry out 
many runs of the parameter estimation. Additionally the use of the automated parameter estimation procedure is recommended to eliminate influence of an operator subjective reasoning [10].

In common practice the random distribution of estimation results is assumed and averaging is used to formulate the final representative modal model. The procedure of formulation of the final modal model is called Modal Model Consolidation (MMC) [2,10,14]. The MMC consists in selection of the most representative estimates of mode shapes out of the set of their available estimates [2]. In [10,11] the authors presented the example of the algorithm of the MMC procedure as well as the recommendation of the model consolidation criteria.

The results of the investigation of modal parameters variability due to manufacturing are rather rarely reported in the literature. An example of such results are presented in [15] for ten specimens of a spot-welded car subcomponent.

\section{Formulation of the problem}

A brake noise and vibrations depend considerably on its components' structural dynamics properties. Designing the brake components that posses appropriate natural frequencies is a technique of minimisation of noise and vibration of the brake.

The brake caliper is a housing for brake pads and pistons. The caliper's motion initiate operation of brakes. The considered caliper was a floating, single piston one made of the cast aluminum A357.0 T6.

A caliper is a path of vibration energy transfer from a disc to a car body during braking, this is a reason of requirements of strictly defined structural dynamic properties of the caliper. The second reason of this requirement is safety of operation since excitation of the structural vibration during braking can cause a fatigue damage of the caliper.

Technology of final production of calipers is based on pressure moulding process in steel moulds, but to build a caliper prototype this process is too costly due to cost of metal mould manufacturing. Rapid prototyping in this case, is the technology used to fabricate physical objects basing directly on CAD data sources. It groups some amount of techniques and machines that allow the fabrication of prototypes in a very short time and with a very low cost. Many different techniques are available on the market, now. The choice of one of them depends on the needs of a customer. As all of them are additive technologies, the main differences between each other are found in the way the consequent layers are built to create parts. Some are melting or softening material to produce the layers (SLS, FDM) while others are laying liquid materials thermosets that are cured with different technologies (SLA, MJM, PolyJet). In the case of lamination systems, thin layers are cut to shape and be joined together. Then rapid prototyping process might be applied to build a mould and gravitational moulding process to produce a prototype caliper, but this prototyping process is different than a final manufacturing process and structural dynamic properties of both calipers, the prototype and the final product, can be different. It is important to possess knowledge about how the application of rapid prototyping technique affects values of the caliper natural frequencies when compared to the variability of the natural frequencies present during the serial production of the caliper.

Thus, apart from investigation of the mentioned analysis techniques, another problem considered in the reported research was to investigate how application of rapid prototyping influences the natural frequencies of the aluminum calipers. The results of such investigation are needed to approve wider application of rapid prototyping in practice of product development in case of calipers.

\section{Application of rapid prototyping technology}

The 3D printing was used in the reported research to build a mould [16]. The technique consists in projecting the liquid adhesive compound on the powder following the shape given by the CAD source, layer by layer. The process contains three steps. First the machine spreads powder. The powder supply piston moves upward and the roller spreads a certain quantity of powder. Then, the jetting head deposits a liquid adhesive compound onto the top layer of a bed of powder object material. The particles of the powder become bonded in the areas where the adhesive is deposited. Finally, once a layer is completed the fabrication piston moves down by the thickness of a layer. The powder supply piston is similar in function to the build cylinder. In this case the piston moves upward incrementally 
Table 1

Comparison between casting parameters used in case of metal mould and in case of RP

\begin{tabular}{lcc}
\hline & Metal mould & RP mould \\
\hline Time of pouring & $6-10[\mathrm{~s}]$ & $6-10[\mathrm{~s}]$ \\
Solidification after: & $115-120[\mathrm{~s}]$ & $10-12[\mathrm{~min}]$ \\
Removing core after: & $200-210[\mathrm{~s}]$ & $40[\mathrm{~min}]$ \\
\hline
\end{tabular}

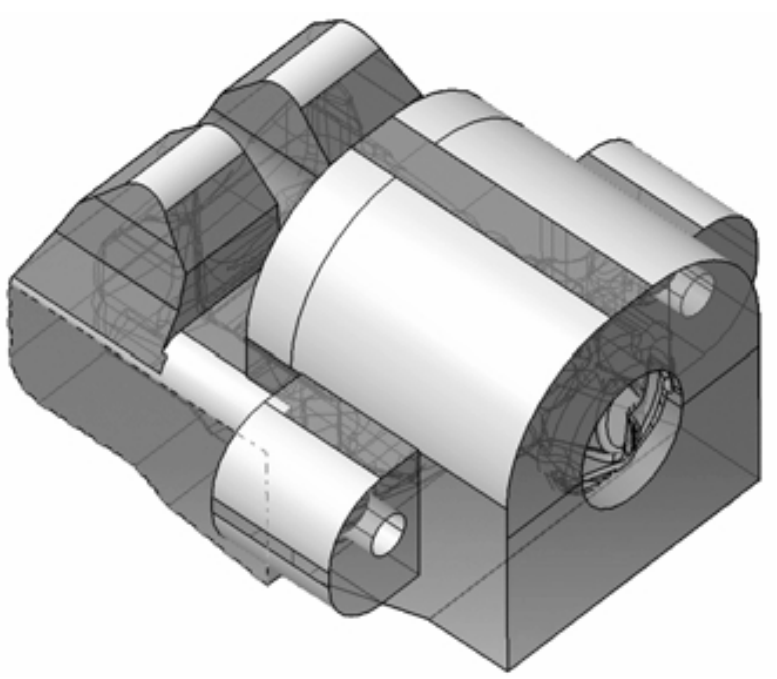

Fig. 1. A CAD draw of the mould.

to supply powder for the process and the roller spreads and compresses the powder on the top of the build cylinder. The process is repeated until the entire object is completed within the powder bed.

The advantages of 3D printing comprise: low cost, technical simplicity, short time of printing and possibility to print elements of very complex shapes. The disadvantages are that the mould is a single use one, a bad surface aspect and often poor static mechanical properties (like: strength, ductility fatigue resistance [9]).

During this research the prototype mould was designed with the use of a CATIA draw of the caliper. The thickness of the mould's wall was between 11 and $13 \mathrm{~mm}$ depending on the region of the mould. As it is shown in the Fig. 1, the mould had three holes. A feeder was added to the biggest one and one gate was added for each hole placed on the fingers. It took 4 hours to print such a mould, but preparation of the metal mould takes the foundry five days. The final shape of the mould is presented in the Fig. 2.

The casting step took place in a foundry. First, the mould was prepared to be poured. The feeder and 2 gates where added in order to avoid thermal shock. It was preheated and then installed in a box filled with metal powder. This powder kept the mould in a vertical position all along the pouring and the cooling phases, but also it made easier the evacuation of the heat. In the Table 1 there is presented a comparison between the parameters used usually during the pouring of a metal mould and the ones corresponding to the prototype mould. The differences of values are explained by the fact that contrary to the metallic mould the sand mold does not conduct the heat flow very well and it takes the metal longer to solidify, what in turn influences the microstructure of the mould. Practically, the grain becomes bigger than the grain in the part obtained with use of the metal mould.

\section{Assessment of the prototype caliper properties}

The investigation comprised assessment of surface aspect, the dimensions, the mass and the internal porosity of the prototype calliper. 
Table 2

List of inaccurately located points (dimensions out of tolerance)

\begin{tabular}{lcccccc}
\hline Point No. & 1 & 2 & 22 & 23 & 24 & 27 \\
\hline Nominal dimension $[\mathrm{mm}]$ & 30 & 20 & 15.6 & 15.6 & 12.5 & 12.5 \\
Actual dimension [mm] & 29.3 & 14.8 & 15.1 & 15.0 & 12.1 & 12.1 \\
Error [mm] & -0.7 & -5.2 & -0.5 & -0.6 & -0.4 & -0.4 \\
\hline
\end{tabular}

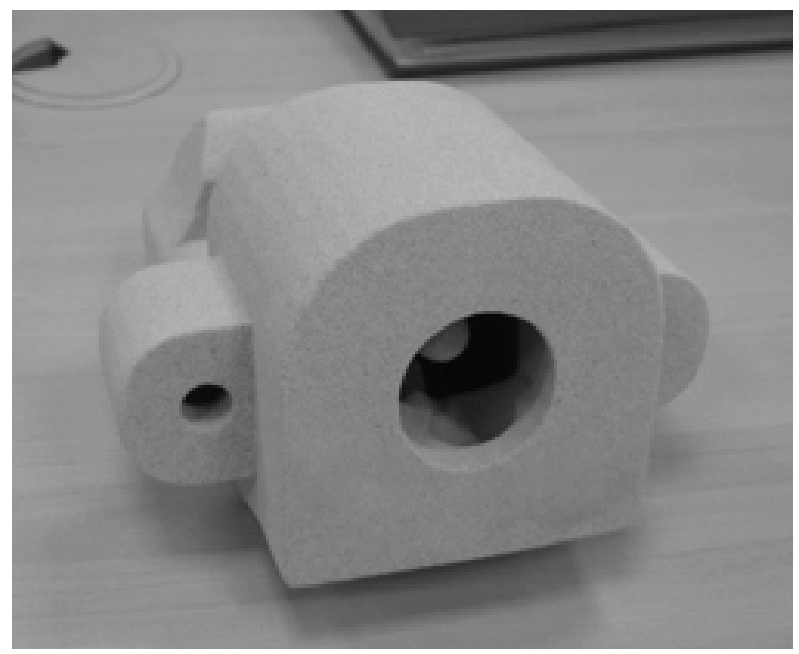

Fig. 2. The mould after cleaning.

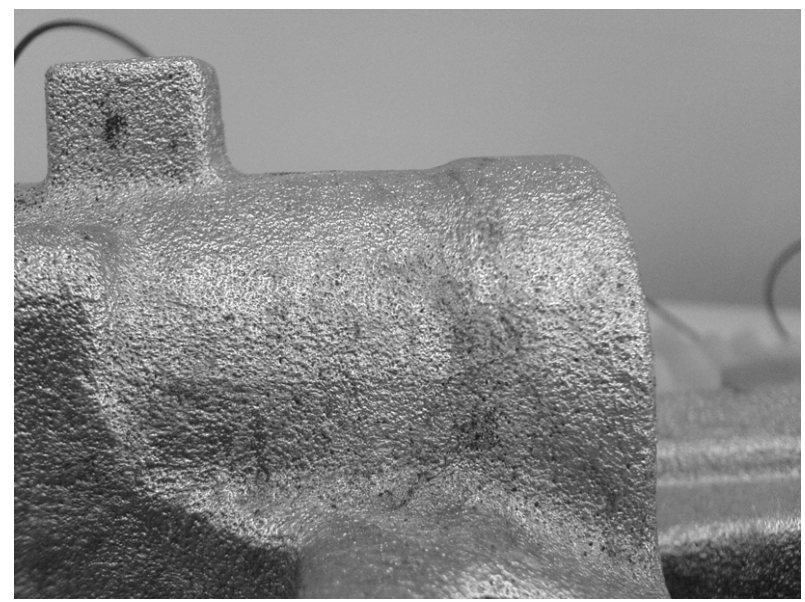

Fig. 3. Illustration of the surface porosity of the caliper produced in the RP mould.

Visual inspection of the surface aspect indicated that the surface of the caliper contains a lot of porosity (Fig. 3). The surface is much rougher on the part produced in the sand mould than the one produced in the metal mould (Fig. 4).

The accuracy of dimensions of the prototype caliper was tested on a measuring coordinate machine. Thirty points were measured (Fig. 5). To be accepted, all the measurements had to be equal to the nominal value with a tolerance of $\pm 0.3 \mathrm{~mm}$. In the Table 2 there are indicated the points ( 6 out of 30 checked) whose position was out of tolerance. The achieved errors were probably caused by: difficult accessibility of points (e.g. points No. from 22 to 27) during cleaning, some left powder, a shrinkage (area of point No. 2).

The caliper produced in the rapid prototyping mode weighed $930 \mathrm{~g}$, and the one from the foundry $964 \mathrm{~g}$. The 


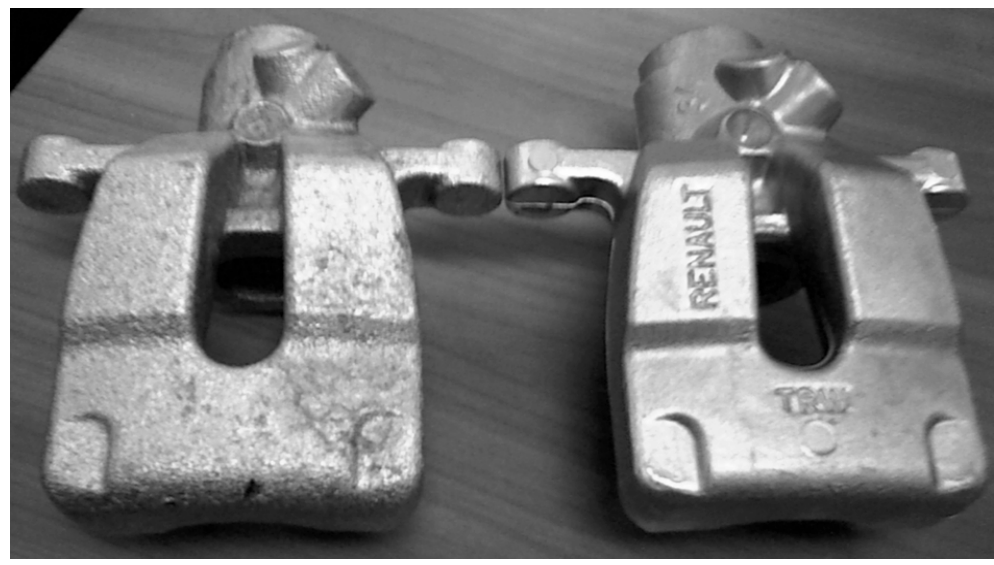

Fig. 4. Comparison of the surface aspect of a caliper from a RP mould (left) and from a metallic mould.
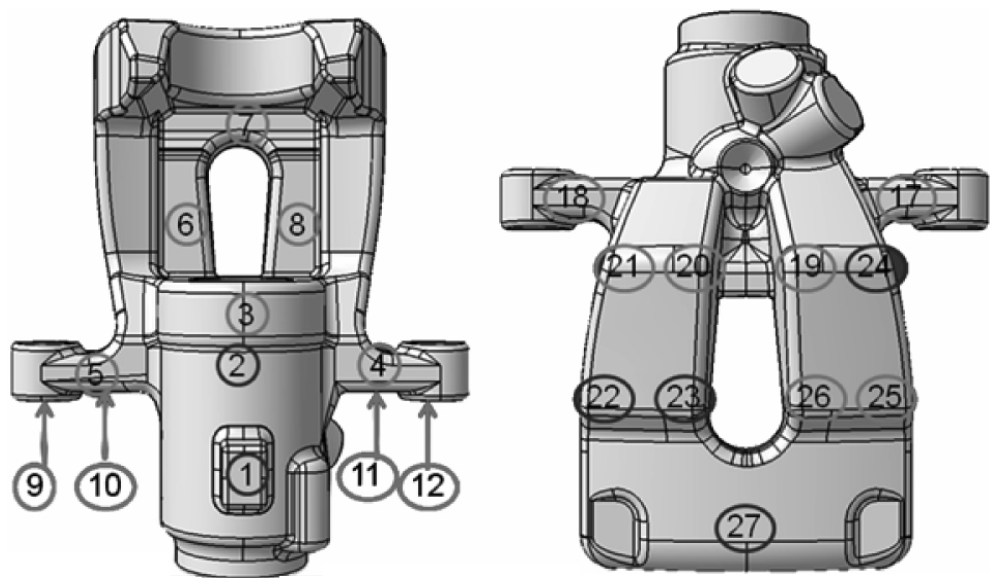

Fig. 5. Visualization of the measured points on the calliper.

difference of $34 \mathrm{~g}$ might have been caused by inappropriate cutting of the feeder off (probably too much material cut off) and/or lower density of material in the caliper produced with RP technology.

Porosity is the most common defect found in Al-Si casting alloys and it is the major cause of rejection of such castings, as it often results in poor mechanical properties such as limited strength and ductility, variable fracture toughness, irregular crack initiation and crack propagation characteristics, as well as a lack of pressure tightness [1, 6]. Two major fundamental effects contribute to the formation of porosity in solidifying Al-Si casting alloys: shrinkage resulting from the volume contraction that accompanies solidification, as well as inadequate liquid metal mobility (bad feeding), as well as gas evolution (mainly of hydrogen) resulting from the decrease in gas solubility in solid metal compared to the liquid one [7,8]. Fatigue is considered to be the most common mechanism by which engineering components fail, and it accounts for at least $90 \%$ of all service failures attributed to mechanical causes of calipers. The internal porosity of the prototype calliper was investigated (only qualitatively) with use of the X-ray radiography. In the Fig. 6 one can see that there are two local areas which contain porosity. The main reason for such porosity was probably the low cooling rate [8].

The results of the carried out assessment indicate that the prototype caliper differs from the one manufactured in serial production. The indicated differences like dimension and mass ones as well as the internal porosity influence the natural frequency values of the prototype calliper. 


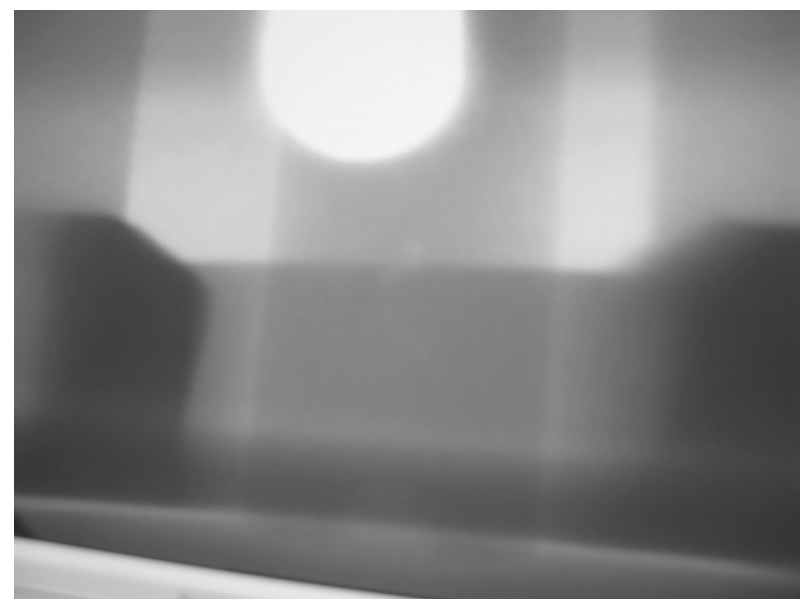

Fig. 6. X-radiography showing the internal porosity at the bottom of the caliper.

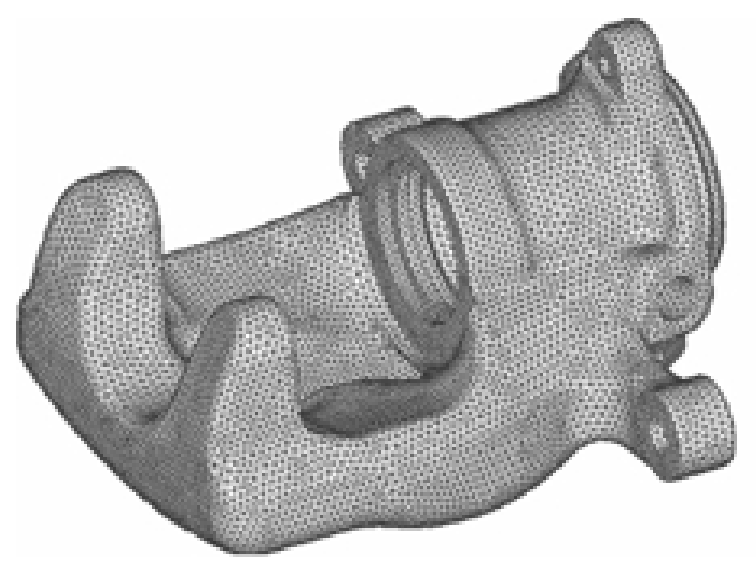

Fig. 7. Mesh of FE model of the caliper.

\section{Numerical study of caliper structural dynamics}

Using FE code a model of the caliper was formulated composed of 240000 tetrahedral 10 nodes elements. The mesh of the model is presented in Fig. 7. The following material properties corresponding to aluminum were assumed: density $\rho=2,7110^{3} \mathrm{~kg} / \mathrm{m}^{3}$, Young's modulus $\mathrm{E}=710^{5} \mathrm{MPa}$ as nominal values. The simulation started with testing of influence of porosity on structural dynamic properties. The porosity location could be found at the places where thermal cooling conditions were poor (bright area in Fig. 8). To simulate porosity of the caliper structure material density has been changed locally in the range indicated by the calipers' manufacturer from $\rho=$ $1.7110^{3} \mathrm{~kg} / \mathrm{m}^{3}$ to $\rho=3.7110^{3} \mathrm{~kg} / \mathrm{m}^{3}$ at selected locations. The results of simulations are presented in the Fig. 9 .

The second important uncertainties which can occur during casting of calipers with use of rapid prototyping technology are variations of dimensions (geometry). The influence of chosen geometrical uncertainties on a natural frequency of the final caliper was tested using sensitivity analysis method implemented in MSC NASTRAN software. The example results are presented in Figs 10 and 11.

The first analysis case dealt with sensitivity of natural frequency values to dimension of the calliper gap (a spacing of the caliper), and in the latter the sensitivity to length of the "finger" of the calliper was analyzed. As it can be noticed from the obtained results, different modes are sensitive in different way to change of dimensions. 


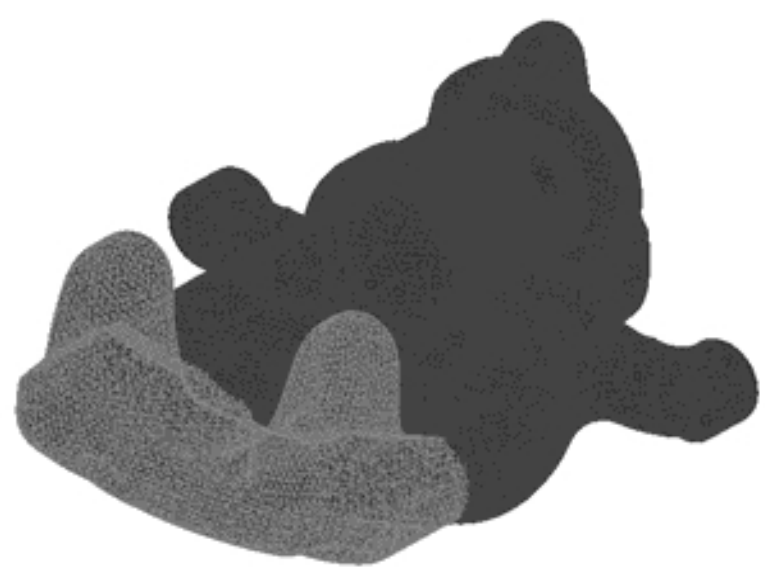

Fig. 8. Location of the caliper porosity volumes.

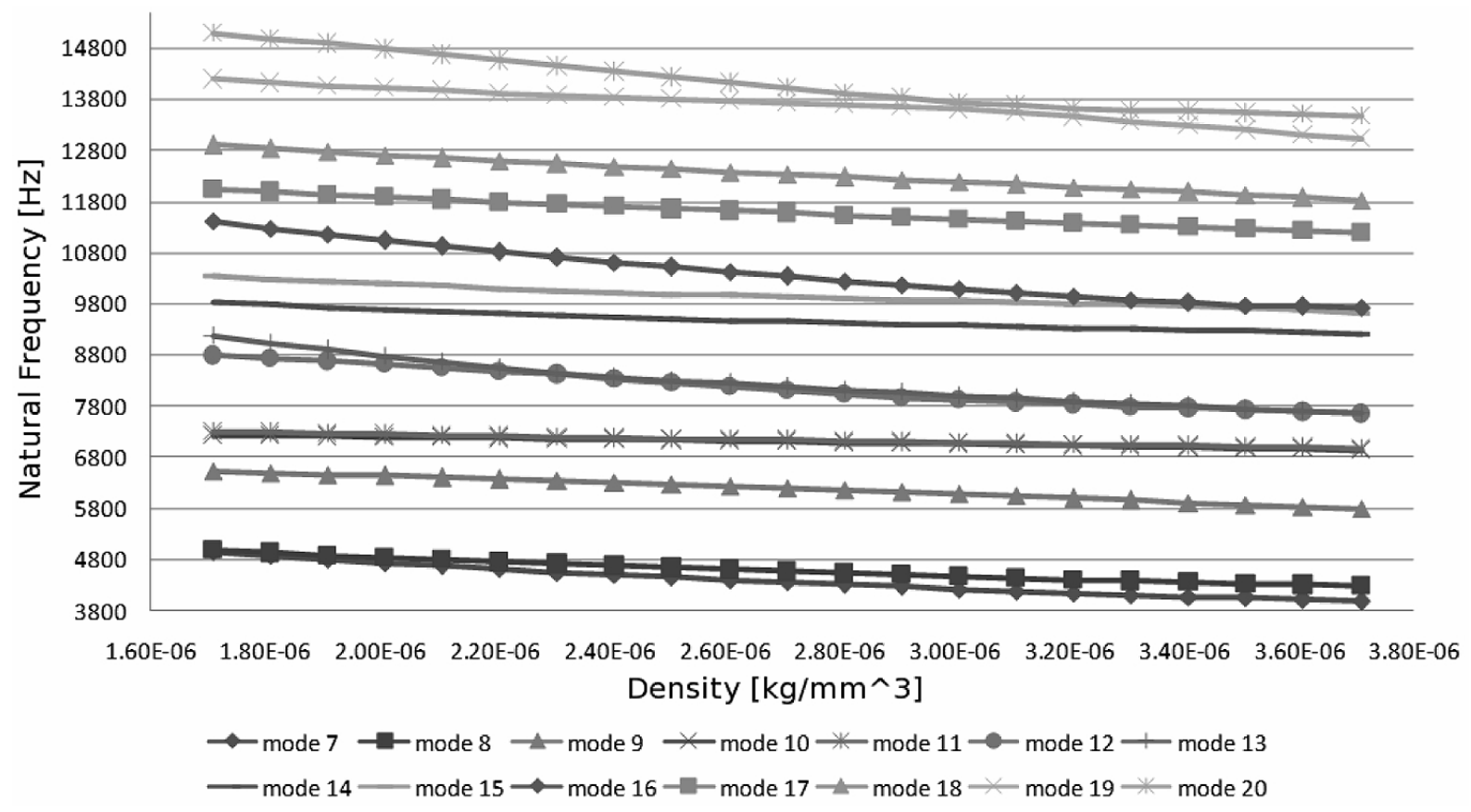

Fig. 9. Sensitivity of the caliper natural frequency to local density of aluminum (porosity).

\section{Experimental assessment of natural frequency variability}

This section reports experiments carried out by the authors with the aim to assess variability of experimental determination of natural frequency. The achieved results were compared with the ones presented in the literature.

Additionally the problem of application of MMC technique in practice was discussed basing on results of assessment of natural frequency variability related to experiment and estimation procedures as well as the variability encountered in manufacturing process.

Finally, the comparison of natural frequencies of the prototype and the serial production calipers was presented.

The reported below results of assessment of variability of natural frequency values of the caliper comprise: variability of experiment (various experimental techniques, exciters, sensors, replacement of sensors, various measuring 

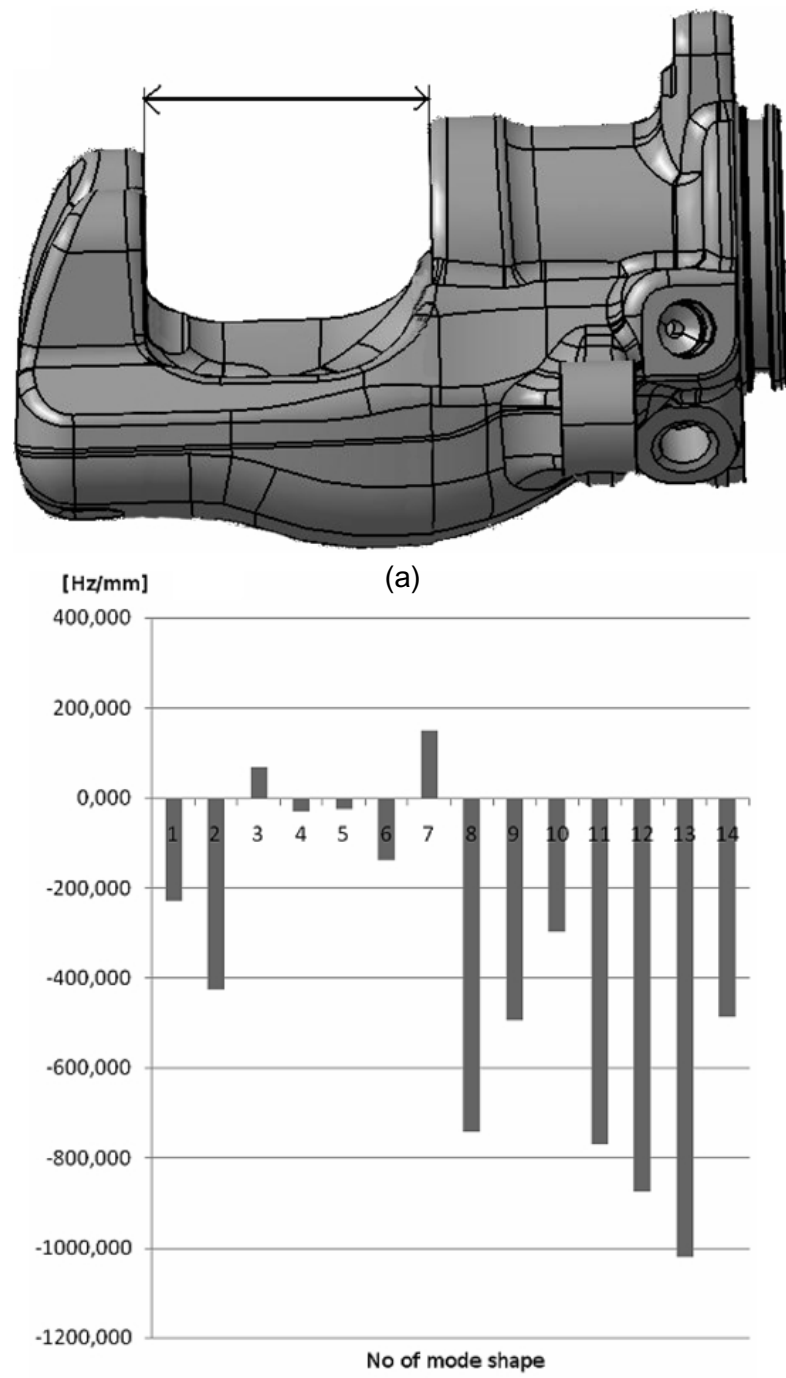

(b)

Fig. 10. The sensitivity of caliper natural frequencies to geometry variation (a spacing of a caliper): a) a view of the caliper b) sensitivity analysis results.

parameters), repeatability of experiments (statistical assessment), variability of parameter estimation (various algorithms and/or their parameters), repeatability of parameter estimation (subjective decision of operators, statistical assessment) as well as variability related to manufacturing process.

First the authors analyzed the experiment data variability. The simulation data (system of 7 degrees of freedom), as well as data measured on a laboratory frame and a disc brake caliper [11] was considered. The investigation of influence of the used frequency resolution on parameter estimation results showed that in case of the simulated data the influence was negligible. Nevertheless, in case of the measured data, the poor representation of a mode shape in the data resulted in larger dispersion of the estimates of the corresponding natural frequencies. The investigation reported in [11] showed that the influence of a modal hammer configuration on natural frequency value scatter was greater than the influence of the used frequency resolution of Frequency Response Functions (FRFs) determination.

The next assessment procedure was the investigation of repeatability of modal analysis results. A set of experiments was performed on the laboratory frame and the disk brake caliper. It was assumed that 29 modal experiments was a satisfactory base to study the results' repeatability. Two sets of modal experiments were carried out. During the second set after each experiment the accelerometers were removed and reattached to the structure. The natural 


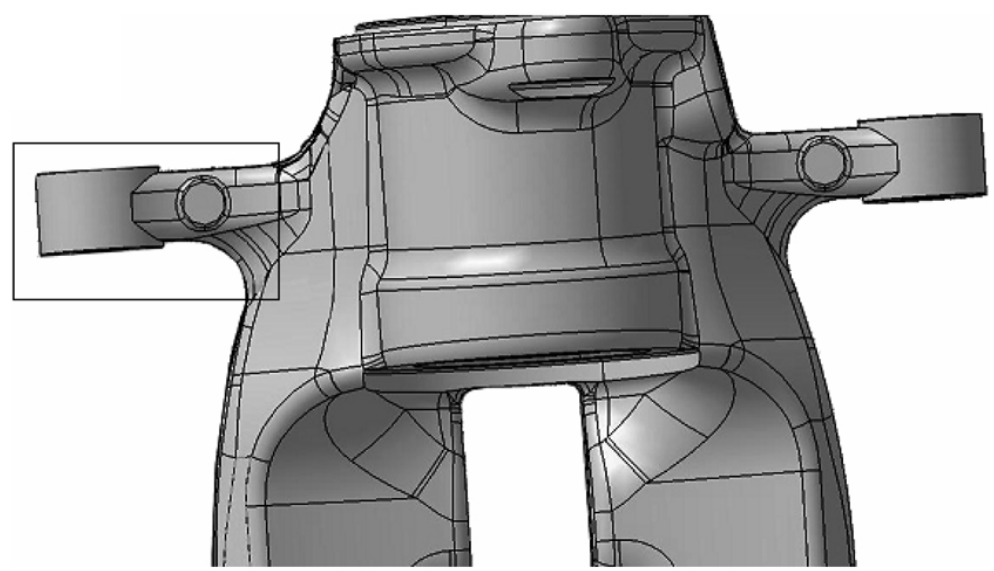

(a)

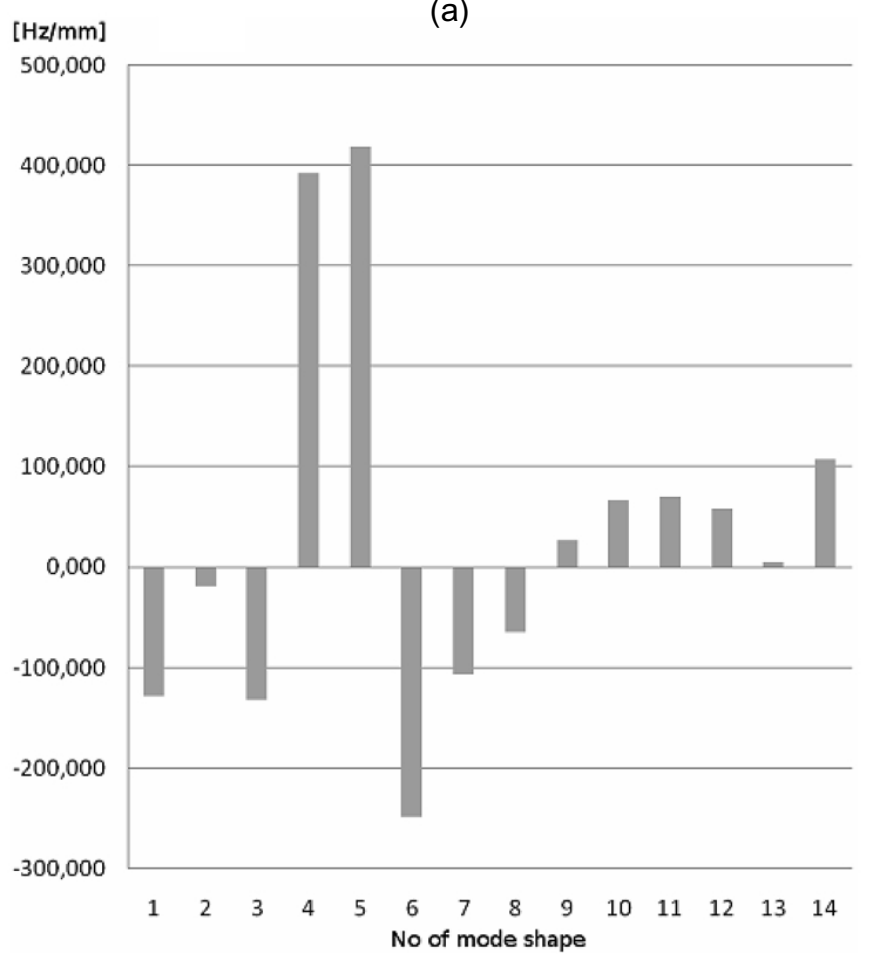

(b)

Fig. 11. The sensitivity of the caliper natural frequencies to geometry variation (a length of finger - dimension indicated by a rectangle): a) a view of the caliper; b) sensitivity analysis results.

frequency value dispersion obtained for the sensor reattachment case proved to be greater for all the estimated mode shapes of the caliper.

A comparison of values of $D_{\sigma}$ obtained by the authors in case of investigation of parameter estimation repeatability as well as variability, and corresponding values reported in the literature are presented in Table 3.

The comparison showed that values of $D_{\sigma}$ varies considerably depending on particular mode shapes. In the worst case (maximum value) the estimated values of $D_{\sigma}$ were in the range of $0.001 \div 0.008$.

To assess manufacturing process variability the authors conducted testing of four cast specimens of the disk brake caliper. A comparison of values of $D_{\sigma}$ obtained by the authors in case of investigation of natural frequency value variability due to manufacturing process and corresponding values reported in the literature is presented in Table 4 . 
Table 3

Comparison of values of relative standard deviation of natural frequency $D_{\sigma}$ due to modal experiment/parameter estimation repeatability/variability [12-15]

\begin{tabular}{lcc}
\hline Modal experiment characteristics & \multicolumn{2}{c}{ Relative standard deviation $D_{\sigma}$} \\
\cline { 2 - 3 } & Minimum & Maximum \\
\hline $\begin{array}{l}\text { Experiment related repeatability - Series of 29 tests, } \\
\text { no sensor reattachment, the caliper [12] }\end{array}$ & 0.00038 & 0.00146 \\
$\begin{array}{l}\text { Experiment related repeatability - Series of 29 tests, } \\
\text { with sensor reattachment, the caliper [12] }\end{array}$ & 0.00085 & 0.00424 \\
$\begin{array}{l}\text { Estimation related variability [13] } \\
\text { Estimation related repeatability [14] }\end{array}$ & \\
Experiment related variability [15] & 0.00193 & 0.00540 \\
\hline
\end{tabular}

Table 4

Comparison of values of relative standard deviation of natural frequency $D_{\sigma}$ related to manufacturing process $[12,14]$

\begin{tabular}{lcc}
\hline Modal experiment characteristics & \multicolumn{2}{c}{ Relative standard deviation $D_{\sigma}$} \\
\cline { 2 - 3 } & Minimum & Maximum \\
\hline Variability - 4 specimens of the caliper [12] & 0.01057 & 0.02371 \\
Variability - 10 specimens [14] & 0.00792 & 0.01171 \\
\hline
\end{tabular}

Table 5

Comparison of values of relative standard deviation of natural frequency $D_{\sigma}$ present in consolidation results estimation related repeatability

\begin{tabular}{lc}
\hline Tested object & $\begin{array}{c}\text { Relative standard deviation } D_{\sigma} \\
\text { Maximum value }\end{array}$ \\
\hline a laboratory frame & 0.00120 \\
a caliper & 0.00531 \\
a helicopter airframe & 0.00465 \\
\hline
\end{tabular}

Table 6

Comparison of values of relative standard deviation of natural frequency $D_{\sigma}$ due to three reasons

\begin{tabular}{lc}
\hline Cause of variability & $\begin{array}{c}\text { Relative standard deviation } D_{\sigma} \\
\text { Maximum value }\end{array}$ \\
\hline Manufacturing related variability & 0.02371 \\
Experiment related repeatability & 0.00424 \\
Estimation related repeatability & 0.00531 \\
\hline
\end{tabular}

The values listed in Table 4 are included in a range of $0.008 \div 0.024$, so all of them are greater than the values corresponding to the experiment/estimation repeatability/variability (compare Table 3). It confirms that EMA is an effective technique of assessment of the natural frequency variability arising during manufacturing process.

The last reported in this paper investigation of the authors dealt with testing of applicability of MMC procedure in presence of experiment results variability. The Table 5 contains values of $D_{\sigma}$ estimated during automated MMC procedure in case of analysis of data resulting from modal testing of the mentioned above three objects (a simulated system of 7 degrees of freedom, a laboratory frame and a disc brake caliper [11]). In the considered case MMC was the automated parameter estimation procedure composed of a set of runs. In each run various part of data and/or parameters of the estimation algorithm were used. The MMC procedure finally averages the estimated modal model parameters. The values listed in Table 5 describe the scatter of natural frequency values corresponding to a single mode shape.

The values listed in Table 5 are of the same order of magnitude as the ones listed in Table 3 . This justifies, in the opinion of the authors, the use of MMC procedure in practice of modal testing. It should be noted that automation of the parameter estimation with use of MMC procedure speeds up the analysis and removes majority of subjective decisions made by operators during the parameter estimation, leading to the more objective results. 


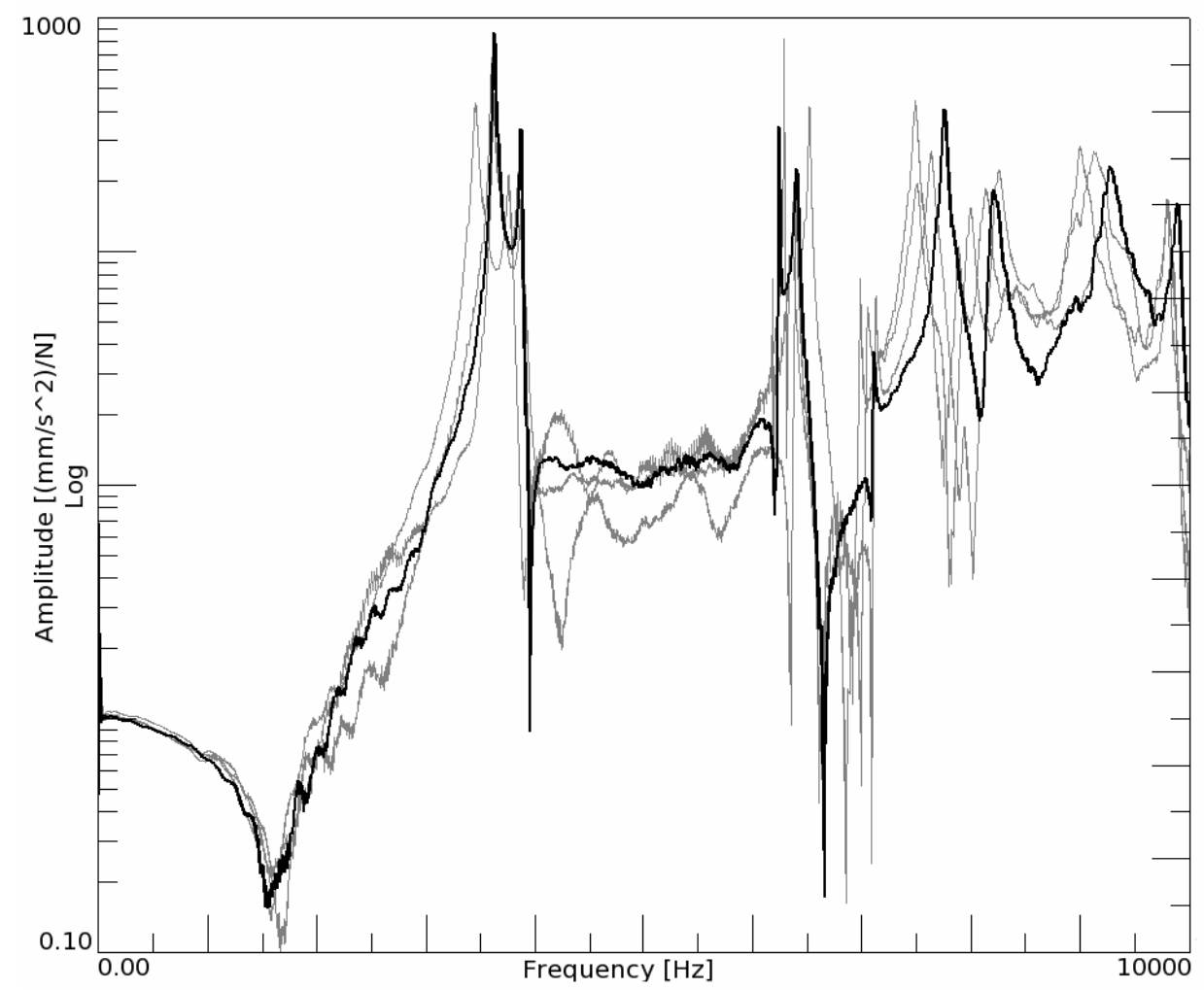

Fig. 12. The results of comparison of FRF measured for calipers obtained using RP mould (thick line) and metal mould (thin lines).

The final comparison of $D_{\sigma}$ values achieved by the authors in the described above three analysis cases for results of testing of the disk brake caliper is presented in Table 6. Comparison of the presented values finally confirms that in case of the considered disk brake caliper the scatter of natural frequency values related to modal experiment repeatability or use of the MMC procedure during parameter estimated is one order of magnitude lower than the scatter related to the manufacturing process.

The carried out procedure of modal testing of the calipers (the prototype caliper and the serially manufactured ones) was consistent with a standard procedure used by a disk brakes manufacturer. During testing the calipers rested on a foam pads to provide boundary conditions close to the free-free ones. For excitation an impact hammer was used. The excitation signal was measured by a force sensor (assembled in the hammer) and the response signal was measured by accelerometers.

In Fig. 12 the comparison of amplitude plots of FRFs measured on the prototype calipers and serially produced calipers is presented. The graphical comparison showed that structural dynamic properties of the considered calipers are qualitatively similar. For quantitative comparison the mean values of the identified natural frequencies of the serially manufactured callipers were calculated. Then, the maximum differences of the natural frequencies from the relevant mean values were calculated and compared to the differences between the natural frequencies estimated for the prototype caliper and that same mean values. The comparison is presented in Fig. 13.

The values compared in Fig. 13 indicated that, at least in the considered caliper case, the application of the rapid prototyping technology did not make the natural frequency values of the prototype caliper differ considerably from the natural frequency values ranges encountered during mass manufacturing process of the calipers. This conclusion supports use of rapid prototyping technique in designing and redesigning of the calipers. The prototype caliper can for example be used during a vibroacoustic testing aiming at verification of computer simulation results without costly necessity of manufacturing of a large amount of calipers.

The authors could use successfully $D_{\sigma}$ coefficient for assessment of variability of natural frequencies, because the values corresponding to variability present during identification of natural frequency values and the ones cor- 


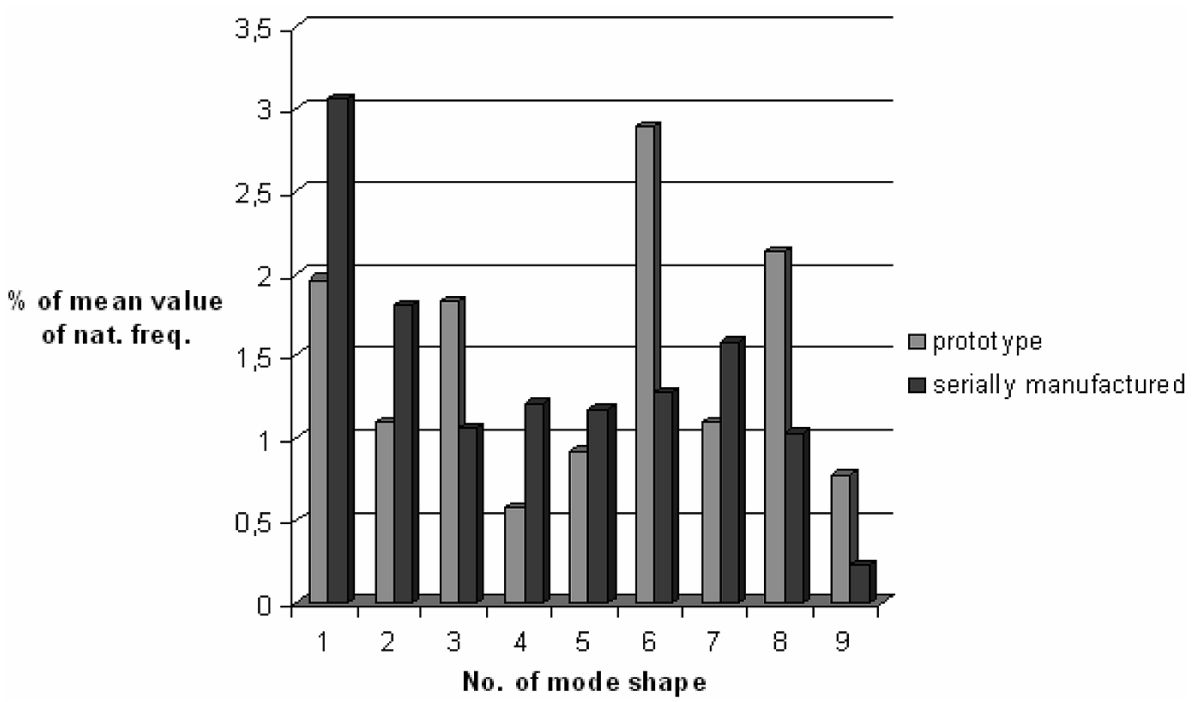

Fig. 13. Comparison of maximum deviation of natural frequencies from the mean value for serially manufactured calipers and deviation of natural frequencies of the prototype caliper from that same mean value.

responding to manufacturing process proved to be of different order of magnitude. The obtained results showed that the values of the coefficient do not depend on the frequency, at least in a simple way. Basing on the obtained results the authors recommend application of $D_{\sigma}$ coefficient in analysis of variability of natural frequencies related to manufacturing process, but in the opinion of the authors determination of the threshold values of the coefficient for decision making purposes still requires further investigations.

\section{Conclusions}

The performed research showed (on the example of a disk brake caliper), that despite some possible disadvantageous properties of the manufactured product, the low cost and fast rapid prototyping techniques are very useful during initial stage of design or during redesign of structural components.

The performed simulation with the use of the Finite Element Method proved its applicability to analysis of causes of natural frequencies variability of mass manufactured objects.

It was confirmed that Experimental Modal Analysis is an effective technique of assessment of the natural frequency variability arising during manufacturing process.

The authors justified the use of the Modal Model Consolidation technique recommended to be used during parameter estimation stage of experimental modal analysis. This technique enables more comprehensive automation of experimental modal analysis what leads to getting more objective results in a shorter time.

\section{Acknowledgment}

The paper is financially supported by the Polish Ministry of Higher Education and Scientific Research under the project CIDAF7 E!4070 PRO-FACTORY Eureka Project.

\section{References}

[1] G.L. Armstrong, Alloy Selection for Automotive Aluminum Castings, SAE Technical Paper, No. 780249, Society of Automotive Engineers, 1987. 
[2] K.S. Chhipwadia, D.C. Zimmerman and G.H. James, III., Evolving Autonomous Modal Parameter Estimation, Proc. of XVII IMAC, SEM USA, 1999, pp. 819-825,

[3] D.J. Ewins, Modal Testing: Theory and Practice, Research Studies Press Ltd., 1985

[4] Y. Govers, M. Böswald, U. Füllekrug, D. Göge and M. Link, Analysis of Sources of and Quantification of Uncertainty in Experimental Modal Analysis, Proc. of ISMA2006, KU Leuven, Belgium, 2006, pp.4161-4173.

[5] W. Heylen, S. Lammens and P. Sas, Modal Analysis Theory and Testing, Katholieke Universiteit Leuven, Departement Werktuigkunde, Leuven, 1997.

[6] J.G. Kaufman and E.L. Rooy, Aluminum Alloy Castings Properties, Processing, and Applications, ASM International, Materials Park, Ohio, USA, 2004.

[7] J.M. Kim, H.W. Kwon, D.G. Kim and C.R. Loper, Jr., Porosity Formation in Relation to the Feeding Behavior of Al-Si Alloys, AFS Transactions 105 (1997), 825-831.

[8] K. Kubo and R.D. Pehlke, Porosity Formation in Solidifying Castings, AFS Transactions 94 (1986), 753-756.

[9] J. Linder, M. Axelsson and H. Nilsson, Influence of the porosity on the fatigue life for sand and permanent mould cast aluminium, International Journal of Fatigue 28 (2006), 1752-1758.

[10] W. Lisowski, T Uhl, P Kurowski, K Mendrok, G. Góral and A. Klepka, Example of Autonomous Parameter Estimation Procedure and its Application to Results of Modal Testing of an Airplane, Proceedings of the ISMA2004, K.U. Leuven, 2004, pp. 1019-1031.

[11] W. Lisowski, Towards a Justification for the use of a Modal Model Consolidation Technique, Proc. of ICEDyn2009 (CD), Ericeira, Portugal, 2009, Paper \#31

[12] M. Luczak, A. Vecchio, B. Peeters, H. Van der Auweraer and L. Gielen, Uncertain Parameter Numerical Model Updating According to Variable Modal Test Data in Application of Large Composite Fuselage Panel, Proc. of ICEDyn2009 (CD), Ericeira, Portugal, 2009, Paper \#25.

[13] N. Maia and J. Silva, eds, Theoretical and Experimental Modal Analysis, Research Studies Press Ltd., 1997.

[14] R.L. Mayes and S.E. Klenke, Consolidation of Modal Parameters from Several Extraction Test, Proc. of XIX IMAC, SEM USA, 2001, pp. 1023-1028.

[15] K. Mendrok, L. Pieczonka and T. Uhl, Assessment of Uncertainty of Experimentally Obtained Modal Parameters, Proc. of ISMA2008, KU Leuven, Belgium, 2008, pp.3899-3910

[16] T. Uhl and G. Nicolas, Influence of the manufacturing process on brackes caliper structural dynamics, Proc. of ICEDyn2009 (CD), Ericeira, Portugal, 2009, Paper \#53. 

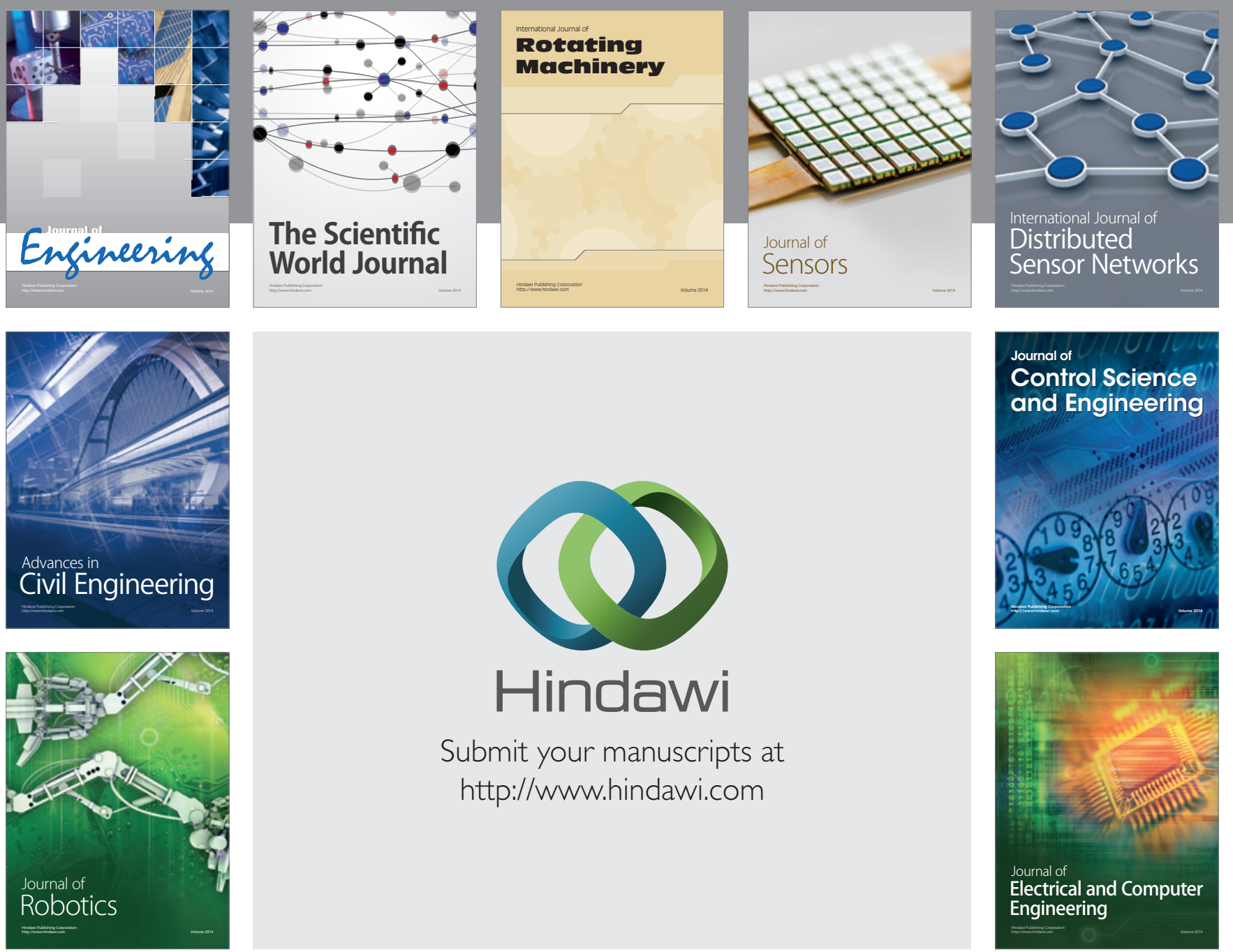

Submit your manuscripts at

http://www.hindawi.com
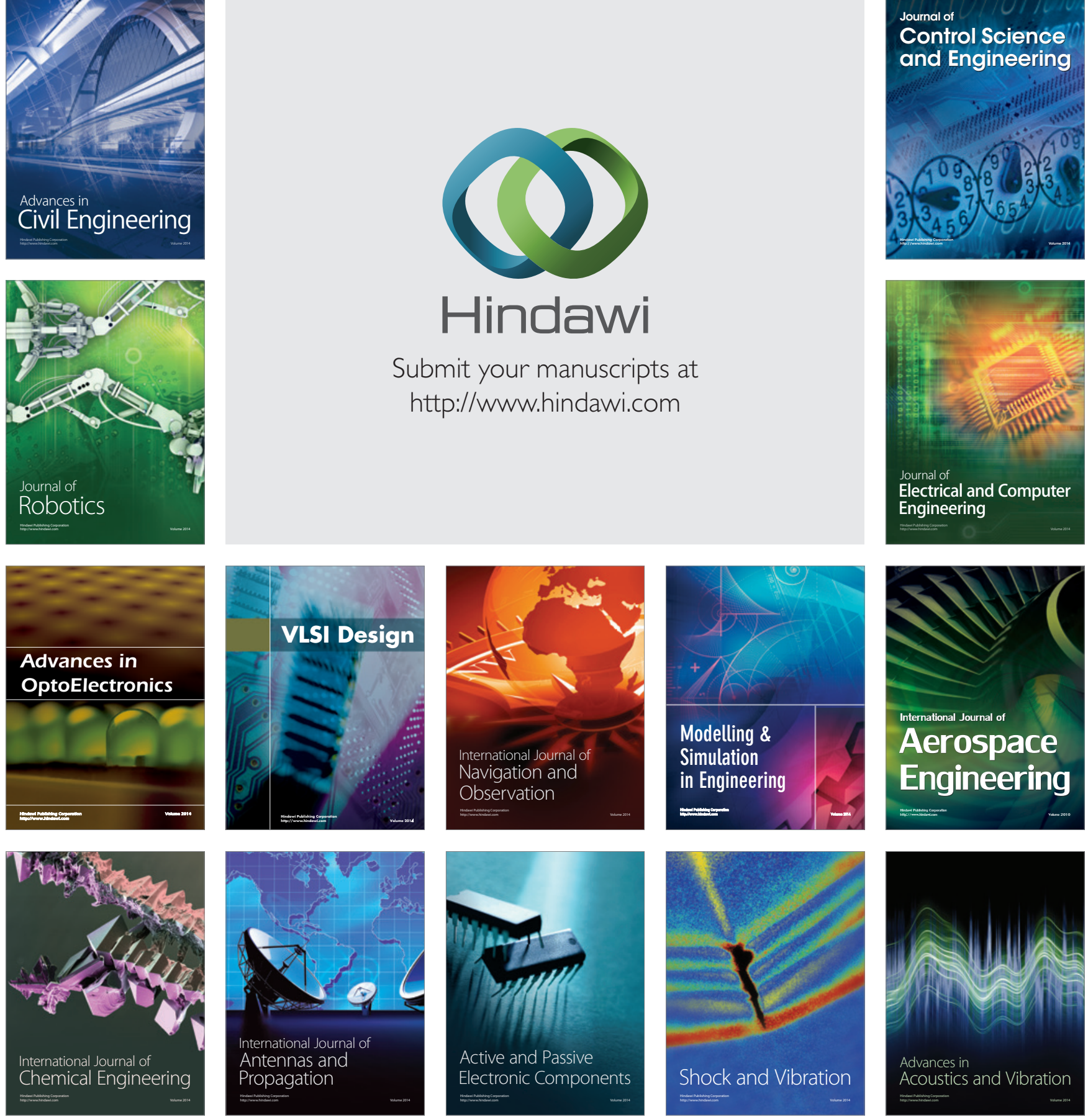